\title{
Los archivos históricos en el siglo XXI: ¿Hacia una redefinición?
}

\section{The historical archives in the $21^{\text {st }}$ century: Towards a redefinition?}

\author{
María del Olmo \\ delolmo.mar@gmail.com \\ Archivo Histórico Provincial de Alicante
}

\section{Resumen}

El presente artículo pretende realizar una mirada, quizás algo filosófica, sobre la realidad actual de los archivos históricos, afectados como el resto de la sociedad, por la revolución tecnológica y de la información. Una mirada que se extiende y proyecta hacia el futuro, tratando de imaginar lo que quizás estamos llamados a ser, sin dejar de contemplar aquellas zonas que van desapareciendo del contorno de nuestra identidad.

\section{Palabras clave}

Archivos históricos; Actividades culturales; Difusión; Archivo electrónico; Archivos y cooperación.

\begin{abstract}
This article aims to take a look, perhaps somewhat philosophical, on the current reality of historical archives, affected as the rest of society, by the technological revolution and information. A look that extends and projects towards the future, trying to imagine what we are perhaps called to be, without ceasing to contemplate those areas that are disappearing from the contour of our identity.
\end{abstract}

\section{Keywords}

Historical archives; Cultural activities; Diffusion; Electronic file; Archives and cooperation 
Recibido: 6/03/2019

Aceptado: $12 / 05 / 2019$

DOI: https://dx.doi.org/10.5557/IIMEI10-N18-069087

Descripción propuesta: Olmo, María del, 2019. Los archivos históricos en el siglo XXI: ¿Hacia una redefinición?. Métodos de Información, 10(18), 69-87

\section{Introducción}

En los últimos tiempos ha sido tratado hasta la saciedad por numerosos autores, y desde múltiples disciplinas, lo que se ha llamado: la revolución de Internet, la revolución tecnológica, la tercera revolución o "la sociedad on line" (Howard, Jones 2005). Se han comparado sus efectos con los de la Revolución industrial, a pesar de que sumidos en esta última revolución que nos arrasa, quizás nos falta perspectiva para el análisis de sus consecuencias.

El mundo de los archivos no es ajeno a esta cuestión y hace años que se encuentra inmerso en una transformación imparable, que afecta en lo más visible a su nueva vida virtual. Pero al igual que para el resto de la sociedad, en nuestro campo también resulta complicado valorar las consecuencias. En tiempos de Internet, de consultas on line y de digitalización de fondos es difícil pensar, por ejemplo, en el futuro físico de los archivos, como casi lo es pensar en el futuro de cualquier otra cosa.

Por último, aunque pueda parecer algo apocalíptico, creo que no debemos perder de vista que la Inteligencia Artificial plantea ya la cuestión de la revisión de la misma profesión. La I.A. en breve tiempo podrá realizar todas las tareas propias del tratamiento documental de la archivística. Creo que a los archiveros solo nos va a salvar lo estrictamente humano, por ejemplo: la capacidad de juicio sobre el valor histórico o no de los documentos, la creatividad, la imaginación y lo que podamos comunicar y difundir del corazón humano que late entre nuestros papeles. (En el caso de las bibliotecas pienso que el futuro es más desolador porque todas las tareas de catalogación son perfectamente robotizables). Hay quizás quien pueda pensar que esto es 
ciencia ficción, sin embargo, a este respecto me ha llamado la atención que la cuestión ya se plantea en los foros profesionales. Anteha Seles, directora del Archivo Nacional de Reino Unido, ha presentado recientemente una ponencia con este título "Los Archivos y la Inteligencia Artificial" al Congreso "El Archivo y documento ante una Nueva Fase de la Revolución Tecnológica". El congreso se celebró en Pamplona entre el 20 y 21 de junio de este año 2019.

Volviendo al presente, la legislación tradicional sobre la materia nos atribuye determinadas finalidades y competencias, que han pretendido siempre describirnos. Por ejemplo, y como marco legislativo común, la Ley 16/1985, de 25 de junio, del Patrimonio Histórico Español nos define así:

Artículo 59

1. Son Archivos los conjuntos orgánicos de documentos, o la reunión de varios de ellos, reunidos por las personas jurídicas, públicas o privadas, en el ejercicio de sus actividades, al servicio de su utilización para la investigación, la cultura, la información y la gestión administrativa. Asimismo, se entienden por Archivos las instituciones culturales donde se reúnen, conservan, ordenan y difunden para los fines anteriormente mencionados dichos conjuntos orgánicos.

Los tratados de archivística clásicos suelen coincidir en la delimitación de nuestras funciones destacando entre ellas las siguientes: reunir, organizarconservar (tratamiento documental) y difundir-servir documentos. Son funciones muy amplias y genéricas que no suelen hablar o contemplar los diferentes contextos en los que deben insertarse las mismas atendiendo, sobre todo, al signo de los tiempos, a un entorno espacio-temporal concreto y en continuo devenir.

Sin embargo, es de justicia reconocer que la puesta al día de las funciones archivísticas en relación con la sociedad de la información e informatizada está siendo realizada satisfactoriamente, en general, tras un gran esfuerzo de adaptación. Es casi obligado poner como ejemplo modélico, casi como arquetipo de los nuevos tiempos de nuestro mundo, al proyecto PARES de la Subdirección de los Archivos Estatales del Ministerio de Cultura y Deporte, en el caso de nuestro país. 
A pesar de todo ello, considero que hay otra revisión pendiente que afecta a algunos aspectos importantísimos de nuestra identidad y que, quizás, hemos ido haciendo casi como reinos de taifas, desde la mejor voluntad de cada uno de nosotros como profesionales. Su conjunto tendría un carácter más global e implicaría un cambio de paradigma en esa identidad. Rompe radicalmente con esa concepción social extensa que nos ha acompañado durante siglos y por la cual se nos ha visto como centros cerrados llenos de papeles acumulados. Territorio reservado a investigadores sesudos, que nos ha entendido como un espacio aislado algo alejado de la ciudadanía. Una estampa que hace décadas que estamos modificando. Se trata de una percepción colectiva que se ha ido desdibujando gracias a la conciencia y al esfuerzo de las últimas generaciones de archiveros, que han querido "abrir las puertas de los archivos" (Olmo Ibáñez 2009) a toda la sociedad y que han encontrado en los últimos tiempos una excelente herramienta en esta nueva sociedad on line. Al adentrarnos en este aspecto se hará imprescindible hablar del amplio concepto de cultura para tratar de encontrar después nuestro sitio allí, en el difuso territorio de la cultura.

Desde el punto de vista reflexivo más general pienso que en esta introducción debo apuntar otro dato sociológico que nos afecta, se trata de la deriva "amnésica" de nuestra sociedad. Aunque no sea tema central en este estudio resulta inquietante la desaparición del hábito de lectura en las nuevas generaciones, las novedosas formas de información que marcan las TICs e Internet caracterizadas por la inmediatez, la reducción de caracteres, la corta existencia vital de la información, arrumbada rápidamente por un ritmo frenético de producción de noticias, etc. El presente, más intenso que nunca, que resta espacio al pasado y que parece que nos aplasta con un efecto de apisonadora desmemoriada. Esta cuestión tiene amplias implicaciones en la futura razón de ser de los archivos, en los históricos y en los que mañana serán históricos. No afrontar esta realidad actual sería grave.

En cualquier caso, esta introducción solo señala unas líneas y el conjunto del artículo no es más que un pequeño esbozo de una mirada concreta sobre este espacio nuestro de los archivos y su estar en nuestro mundo. 


\section{Memoria democrática}

Creo que se trata de una cuestión que necesita un apartado propio y casi exige comenzar por ella porque hoy, insertos en un Estado democrático y de derechos, es razón de ser de nuestra existencia. Un antes y un después, un hito histórico en nuestro devenir lo marco la Ley 52/2007. Considero que desde la Ley 52/2007 conocida como "Ley de la Memoria Histórica" los profesionales de los archivos tenemos una especial responsabilidad derivada y enmarcada en ella. Es la responsabilidad de contribuir a que las víctimas puedan ejercer sus derechos, y contribuir también a favorecer la investigación y difusión de toda la documentación que preservamos sobre la materia. En los últimos años hemos hecho todos un esfuerzo enorme en este sentido, pero me gustaría plantear qué más podemos hacer. Se desprende de ello cuestiones como los plazos de acceso, la Ley de Secretos Oficiales y tantos otros aspectos que han estado debatiéndose por la opinión pública recientemente a través, por ejemplo, de distintas noticias aparecidas en los medios de comunicación.

Quiero también incidir en que creo que los fondos afectados por la Ley $52 / 2007$ deben ser prioritarios en nuestros calendarios de descripción documental, si queremos responder a esta demanda de derechos de la sociedad.

\section{Una nueva identidad: Centros culturales}

La autoría de la palabra "cultura" se atribuye a Cicerón y etimológicamente procede del verbo "colo" que se refiere al cultivo en la agricultura, Cicerón lo transforma convirtiéndolo el cultivo del alma. Trasladándolo al ser humano lo traduciríamos como el cultivo de uno mismo. En su sentido más amplio y común seguramente entendemos a la cultura como la transmisión social de conocimientos que constituyen un patrimonio común.

Retomando lo que quería decir en el punto anterior sobre memoria democrática, últimamente se ha vinculado el término cultura con el adjetivo "democrática" conformando el par "cultura democrática". Esta pareja o esta asociación se entiende también como sinónimo de ciudadanía y tiene como 
objetivo construir una cultura que recoja, desarrolle y eduque en el conjunto de los valores del sistema democrático.

Si los archivos queremos ser agentes activos en este terreno, condición que nos es propia por nuestra función esencial de memoria social, y terreno con el que estamos obligados como servicio público que somos, creo que hay tres rasgos que nos conforman y que tenemos que ayudar a dar a conocer. Somos lugares de derechos, historia, y memoria. En esa labor de difusión de nuestra condición democrática se insertan muchas de las nuevas actividades que van reconformando el perfil del archivo transformándonos en lo que se conoce comúnmente como centro cultural. Voy a dedicar unas líneas a algunas de ellas.

\subsection{La difusión de quiénes somos y para qué servimos}

\section{Didáctica}

En esa condición de centros de cultura democrática cobra un papel especialmente relevante nuestro trabajo en el ámbito de la didáctica. La atención al sector de la ciudadanía en formación académica me parece esencial si queremos contribuir a construir una sociedad consciente de nuestro papel y de la labor que nos corresponde hacer en ese sentido. La principal herramienta que tenemos para desarrollar este objetivo es la didáctica sobre archivos.

Dentro este ámbito de la didáctica en archivos constituye un referente importante las Jornadas "Educación y archivos" del Institut de Ciències de l'Educació (ICE) de la Universistat de Barcelona, que ya van por la sexta edición y que han creado un foro de debate común entre universidad y archivos. En la última edición se han propuesto como objetivos los siguientes:

a) Reflexionar sobre el papel de los archivos como instituciones que favorecen la creación de conocimientos por parte de los alumnos.

b) Aportar elementos para hacer presentes los archivos en el mundo educativo en tanto que instituciones abiertas al servicio de la ciudadanía. 
c) Difundir estrategias y recursos pedagógicos relacionados con el uso didáctico de los documentos.

d) Promover la interacción entre docentes y archiveros, por tal de favorecer la búsqueda relacionada con la aplicación didáctica de los fondos de archivo con el objetivo de contribuir a la mejora de la cualidad de la enseñanza.

La didáctica requiere, casi imprescindiblemente, un trabajo en colaboración con los profesionales de la enseñanza, algunos de ellos nos llevan dedicando atención en su trabajo académico desde hace años. Se puede citar como ejemplos de los docentes a Gemma Tribó con su libro "Enseñar a pensar históricamente: los archivos y las fuentes documentales en la enseñanza de la historia" (2005) o a Jesús Giménez y su artículo "El archivo en la enseñanza de la Historia" (1995). Nosotros también comenzamos a estudiar "académicamente" este aspecto, pido disculpas por la autocita, Consuelo Poveda y María del Olmo "La didáctica en el AHPA". (2014, p. 235 y ss.). Pero como señalan Melero y Cruces en su artículo "Los archivos y los programas pedagógicos" (1991 vol. 41, no 2, p. 33-51), el trabajo en equipo todavía es una asignatura pendiente:

La relación entre Archivo y educación debe establecerse a través de los Centros de enseñanza y sus temas de estudio. Es, por tanto, primordial el trabajo conjunto entre archiveros y docentes, remediando así la carencia de comunicación existente entre las fuentes de la Historia y la docencia. El archivo, generalmente, no ha participado en la función educativa o, al menos, en menor medida que las bibliotecas y los museos; sin embargo debemos recordar que, los archivos, son la memoria ordenada y coherente de la Historia.

En este sentido resalto la experiencia tan interesante que el Archivo Histórico Provincial de Alicante ha compartido con el Centro de Formación de Profesores (CEFIRE) de la GVA, en las jornadas "Herramientas para el trabajo de la memoria democrática en las aulas valencianas", que ha tenido 3 ediciones distribuidas por Alicante, Valencia y Castellón en 2018.

\subsection{Memoria de la sociedad: exposiciones, marketing, diversidad de actos culturales}


Este apartado se refiere y contempla la difusión en toda su diversidad y potencialidad posible, intenta abarcar la perspectiva más amplia.

Para comenzar quiero decir unas palabras sobre la importancia de nuestra presencia en los medios de comunicación en cualquiera de sus formatos posibles. Ellos están en el centro del debate social y marcan muchas veces la actualidad. A nosotros nos abren puertas insospechadas en el camino de situarnos también en el centro del ágora pública y pueden convertirse en unos grandes aliados para la difusión de nuestra actividad. La dedicación a la elaboración de notas de prensa de nuestras actividades, la insistencia en ser noticias y nuestra presencia en entrevistas de radio y televisión, por ejemplo, deben estar presentes en nuestro horizonte laboral como recursos, como herramientas en el ámbito de nuestra difusión.

Si nos adentramos, ya más específicamente, en lo que es y debe ser nuestra labor propia de difusión podemos comenzar recordando a la Fundación Sierra Pambley y a una de sus interesantes jornadas, la que dedicó precisamente al tema con el título: $<<3^{a}$ Jornada Archivando: "La difusión en archivos" $>>$ (González Cachafeiro 2010) por la perspectiva tan amplia en torno a la difusión que abordó: 


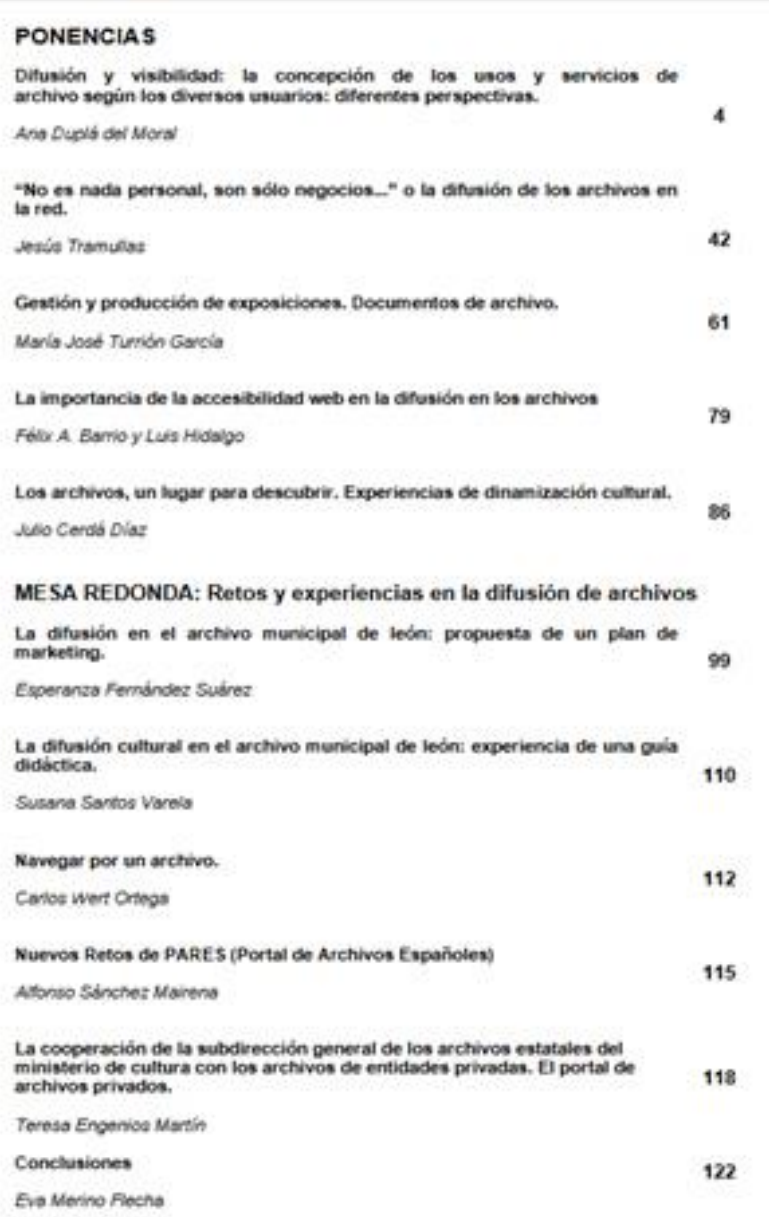

Recomiendo su lectura detenida. Voy a tratar de plantear algunas cuestiones para la reflexión sobre actividades que se han convertido en tradicionales para la difusión y otras que no los son tanto.

\section{Las exposiciones}

Las exposiciones ya conforman nuestra realidad más cotidiana independientemente del tamaño de nuestros archivos y de nuestra adscripción administrativa, nos hemos lanzado a ellas con plena conciencia de su interés y de su potencialidad desde hace años. Así, como elemento fundamental de la difusión en archivos hace décadas que forman parte de nuestras tareas. Las muestras documentales nos permiten ofrecer nuestros fondos a un público mucho más amplio que el que nos visita con fines de investigación.

Las exposiciones tienen vocación de llegar a toda la ciudadanía y son un excelente reclamo para los centros escolares, que comienzan a incluir a los 
archivos en su programación anual de visitas culturales en su entorno. Pero me interesa señalar que esta vocación globalizadora, de convocar a toda la sociedad tiene aspectos menos explorados que se están mostrando muy eficaces para darnos a conocer y que favorezcan el que esta sociedad nos sienta como suyos. Las experiencias analizadas de apertura del espacio expositivo a instituciones públicas y privadas y a particulares para la realización de muestras de carácter colaborativo en las que el centro de acogida aporta la parte más importante en gestión y composición de fondos a exhibir ofrecen un resultado muy positivo. El nivel de implicación en el proyecto de personas y entidades externas es muy alto, la generosidad en la cesión de fondos es enorme y ellos mismos aportan público nuevo a los centros. De este aspecto se deriva una consecuencia interesante e inesperada. Se trata de la donación de documentos por los particulares colaboradores.

La aproximación a un archivo histórico con este nivel de participación conlleva el despertar de una sensibilidad especial hacia nuestro trabajo y la función que desempeñamos en la sociedad generando, en muchos casos, el deseo de contribuir con documentos de archivos familiares cuyo destino futuro suele ser incierto. Las donaciones constituyen una vía de recogida de fondos muy importante para los archivos históricos públicos, ya que aportan documentación que viene a completar espacios documentales que como depositarios de documentación de la administración pública nos son ajenos.

Es la historia privada, lo que Unamuno llamó la intrahistoria y las historias biográficas, que hacen cobrar especial vida a nuestros archivos, de alguna forma se puede afirmar que nos humanizan. Recientemente hemos asistido al debate entre historiadores sobre el par Historia/Memoria, sin entrar en él, lo cierto es que el ámbito de la memoria y la historia que conservamos se amplía de este modo considerablemente. Es por esto que otro centro de nuestra dedicación debería ser, quizás, la realización de campañas de recogida de fondos particulares. Podríamos añadir en este punto otro detalle a valorar, el estudio del mecenazgo en archivos, cuestión bastante normalizada en el campo de los museos.

Sin embargo, un aspecto sobre el que creo que habría que llamar la atención en este apartado es el de la formación académica en el ámbito de exposiciones, las exposiciones no pueden ser sin más una muestra de documentos en 
vitrinas. El estudio de este campo de desarrollo cultural colectivo está siendo objeto de gran interés en muchos campos y casi exige una preparación formativa de carácter monográfico. Si queremos ahondar un poco más en lo que está sucediendo en dicho ámbito me parece muy relevante y sugerente el foro conformado por lo que se ha denominado "Historia pública" y que cobra presencia activa de forma imparable. Su eje principal sería la labor colaborativa de todos los agentes sociales implicados para hacer presente la historia en la vida social. El National Council on Public History define la "Historia pública" como un movimiento, una metodología y un enfoque que promueve el estudio y la práctica en colaboración de la historia; sus practicantes asumen en la misión de que sus particulares puntos de vista sean accesibles y útiles para el público", en la cuestión de la accesibilidad se vincula la historia pública con la historia digital. En este aspecto el campo de la "Historia pública" presta atención especial a las muestras o exposiciones sobre historia haciendo hincapié en la incorporación de elementos interactivos, audiovisuales complementarios, introducción de objetos museísticos, recreaciones de espacios históricos, rutas históricas, etc. Como ejemplos de actividades sobre "Historia pública" se pueden poner los casos de Italia con la reciente Seconda Conferenza Nazionale di Public History AIPH, o el caso americano con el Annual Meeting Council on Public History (Indianapolis 19-22 de abril de 2017).

Por último, y ya que he mencionado las rutas históricas, no quiero dejar de señalar la importancia que puede tener el integrarnos dentro de lo que se ha denominado "Circuitos de turismo cultural" muy en boga en este momento. Estos circuitos deberían incluir en sus rutas las visitas a nuestras exposiciones.

Para cerrar el tema de las exposiciones hago una consideración final. Dada la condición efímera de estas por su misma naturaleza, me parece de interés la grabación de una audiovisual de cada una de ellas. De esta manera, y por medio de dicho recurso, nos queda de ellas un testimonio documental en un formato que nos permite preservarlas en el tiempo.

\section{El márketing}

Parece un concepto muy amplio y muy extendido. La RAE remite marketing a "mercadotecnia" y la define así: 
mercadotecnia

De mercado y -tecnia.

1. f. Econ. Conjunto de principios y prácticas que buscan el aumento del comercio, especialmente de la demanda.

Sucintamente el marketing más extendido sería el conjunto de herramientas que nos den visibilidad y que trasladen a la sociedad información de nuestro "valor de uso": cartas de servicios, páginas webs, redes sociales, publicidad, presencia en prensa, presencia en foros diversos, etc. Pero quería referirme también a otro aspecto del marketing menos explorado, el de la venta de productos relacionados con nuestra actividad en nuestros mismos centros. Las tiendas de los museos (un referente puede ser el Museo del Prado) se han convertido en una parte importante de sus espacios. Además, por ejemplo, en la Biblioteca Nacional también existe una tienda en el hall, cerca de sus salas de exposiciones, y se pueden adquirir sus catálogos, otros libros y algún producto de otro orden. Las exposiciones de los archivos pueden ser objeto de la elaboración de un merchandising que contribuyera a nuestra publicidad y difusión. Al mismo tiempo, también podía generar otra vía de ingresos: catálogos, carteles de las exposiciones, reproducción de documentos relevantes: fotografías, planos, etc.

\section{Diversidad de actos culturales}

Cada vez están más presentes en la oferta de actividades: presentaciones de libros, conciertos, recitales de poesía, conferencias, jornadas y congresos, etc. En muchas ocasiones estos actos no tienen que ver directamente con nuestra actividad, pero son una buena forma de darnos a conocer y una fuente de atracción de potenciales nuevos usuarios. En cualquier caso si que quiero incidir en aquellos actos que tienen que ver con nuestra presencia científica en la sociedad y en el ámbito académico. La organización de eventos de carácter profesional: jornadas, congresos, etc., tanto para contar a qué nos dedicamos, como para difundir fuentes documentales para la investigación. En este aspecto la relación con la universidad será importante. Profundicemos un poco en ella.

\section{Hermanamiento en la Universidad}


Nuestros más antiguos, tradicionales y fieles clientes han sido los investigadores provenientes de la academia. No siempre las relaciones han sido óptimas con ellos, pero en la medida que los archivos han ido ganando en apertura también se ha ido incrementado la sintonía entre nuestros dos ámbitos. Sin embargo, cuando hablo de hermanamiento quiero plantear otros aspectos distintos. Me refiero a la condición de los archivos como centros de investigación. Esta condición puede dar lugar al establecimiento de proyectos de investigación compartidos. Superaríamos así nuestro carácter único de "gestores documentales" y nos podríamos implicar como "investigadores" en esos proyectos optimizando nuestros conocimientos sobre un determinado conjunto documental, derivado de nuestro trabajo profesional, y poniéndolos a disposición del dicho proyecto.

También quiero plantear la cuestión de la presencia de archiveros en la formación universitaria, en los grados de ciencias sociales y en los de formación del profesorado de dicha área, en todos los niveles: enseñanza primaria, secundaria, bachillerato y universidad. Nuestra colaboración en forma de charlas, participación como profesores asociados, etc. puede tener un rédito inusitado. Ellos son los encargados en formar a las nuevas generaciones de la sociedad, si logramos despertar la conciencia de que somos la memoria y la historia de dicha sociedad y de nuestro papel activo en el corazón de los docentes del futuro, las repercusiones a largo plazo pueden ser muy importantes. Además considero que aportamos una faceta al programa formativo que complementa y enriquece los planes de estudio.

La firma de convenios de prácticas de estudiantes universitarios entre archivos y universidades abre la vía de nuestra aportación a la formación universitaria práctica, muy presente desde el Plan Bolonia en los grados universitarios. Por un lado, suponen incorporar un nuevo papel en las funciones del archivero, la actividad formativa, y por otro lado están aportando una ayuda interesante a plantillas normalmente muy reducidas, con proyección de futuro, ya que formamos a potenciales nuevos archiveros.

Por último, algo más extendido y factible. La programación conjunta, archivos/universidad, de congresos y jornadas sobre historia, memoria, fuentes documentales para la investigación, etc. enriquece especialmente el desarrollo de todos estos eventos. 


\section{Cambios internos}

\section{El lenguaje de los archivos}

Decía Ortega y Gasset que la claridad es la cortesía del filósofo, en realidad esa claridad debe ser la cortesía y la obligación de cualquier profesional cuyo trabajo vaya destinado a la sociedad. Es el deber que tenemos de hacernos inteligibles por el público al que nos dirigimos, tratando de evitar "jergas internas", argots profesionales, que nos hagan indescifrables. Llama la atención encontrar entradas de blogs de profesionales como esta de Julián Marquina que me ha sorprendido: "Palabras que toda persona debe interiorizar para entender a su bibliotecario". Se ha hecho un esfuerzo ímprobo por parte de la comunidad internacional de archivos en las últimas décadas, para encontrar un lenguaje homogéneo y común, para establecer, por ejemplo, una normativa internacional. Era una asignatura pendiente trascendental en la que las bibliotecas nos llevaban muchísima delantera. También nos hemos puesto como objetivo crear webs y bases de datos claras, intuitivas y navegables, que recojan todos los acuerdos internacionales en nuestro ámbito.

Sin embargo, quizás nos falta conseguir esa misma claridad en conceptos y terminología archivística, que haga innecesario un intérprete que nos traduzca al usuario común, exagerando muchísimo. Desde luego es una demanda bastante frecuente entre los investigadores y creo que nos debería hacer pensar.

\section{Reequilibrio de espacios}

Nuestros hermanos bibliotecas y museos, agentes de cultura con visibilidad social, llevan siglos abriendo sus puertas a toda la ciudadanía, aprendamos de ellos y abrámonos al público amplio. La pregunta previa a formular sería a quiénes queremos dirigirnos. 
En la actualidad es una realidad incontestable la existencia en un mismo archivo de dos espacios: el físico y el virtual, el segundo es ya tan real hoy como el primero. El segundo necesita de una construcción virtual tan exigente casi como el propio edificio físico. Considero que el espacio virtual debe ser cuidado con el mismo esmero que lo hacemos con el espacio físico, ya que en muchas ocasiones constituye nuestra carta de presentación, la primera forma que el usuario tiene de acercarse a nosotros. Nuestra web o nuestras redes sociales: Facebook, Instagram, Twitter, canal de YouTube etc. son importantísimas puertas a la sociedad hoy, necesitan atención constante, actividad permanente y puesta al día frecuente. Contar con expertos en redes sociales que nos asesoren puede mejorar nuestro trabajo en la red.

En el espacio físico quizás se hace más patente la necesidad de reequilibrio. La parte tradicional destinada a difusión está ya muy contemplada en nuestros archivos. Hay que indicar que desde 1992 existe una publicación del Ministerio de Cultura y Deporte sobre "Recomendaciones para la edificación de archivo" escrita por Julio Enrique Simonet y bien conocida. La mayoría de los centros cuentan con espacio expositivo y salón de actos ${ }^{1}$. Quizás la cuestión pendiente sea la que tiene que ver con la didáctica, la existencia de aulas didácticas es menos frecuente, sin embargo la experiencia demuestra que la inversión en un espacio destinado a los centros escolares aporta unos resultados a corto, medio y largo plazo importantísimos. En las salas de exposiciones quizás lo que resulta más necesario y se convierte en objeto de atención y estudio a considerar es su reconversión para convertirse en espacios realmente interactivos en los que también sea posible incorporar ciertos detalles museísticos para recreación de contextos.

No se puede olvidar una realidad, las salas de consulta física están sufriendo una profunda transformación en el número de usuarios. Las estadísticas empiezan a inclinarse hacia el lado de las consultas on line y ese es el futuro a más o menos largo plazo. Es una reflexión importante, que deberíamos empezar a hacernos.

\footnotetext{
${ }^{1}$ La cesión del salón de actos a instituciones públicas y privadas es otro camino para obtener visibilidad y darnos a conocer con efectos también muy positivos.
} 


\section{Profesionales: transversalidad y trabajo en equipo}

Pienso que la actual transversalidad de muchos de nuestros proyectos hace ya indispensable la inclusión de otras disciplinas en nuestras plantillas de personal. Algunos perfiles profesionales diferentes del archivero son clásicos, en muchos casos ya como parte de los equipos, en nuestros centros o en asociaciones puntuales y temporales para nuestro trabajo. En el primer caso un buen ejemplo son los restauradores, y para el segundo los arquitectos, (no puedo olvidar la gran labor de Juan de Herrera arquitecto del archivo de Simancas, en colaboración con el archivero y el rey y que recogí en el artículo "El latido del corazón de Simancas", 2010). Más recientemente los informáticos se han incorporado con normalidad a nuestro trabajo diario y quizás sea importante contemplar para los grandes archivos la inclusión en sus plantillas de técnicos en exposiciones y de algún especialista en didáctica.

Los especialistas en recursos humanos y técnicos de gestión de personal en los últimos tiempos no hacen más que recordar la importancia fundamental del trabajo en equipo y del conocimiento de las potencialidades de cada uno de los miembros del mismo, para construir un engranaje fecundo y optimizar al máximo el rendimiento. Para los cargos directivos sería muy importante cierta formación en estos aspectos.

Reconozco que este punto constituye una reflexión abierta que implica valorar si podemos abarcar todos esos ámbitos, por ejemplo y sencillamente, con una formación mayor en ellos de los propios archiveros. Es fundamental en este campo ser muy realistas a la hora de tener en cuenta el coste económico que implicaría.

No quiero dejar de señalar que, para esta óptima eficacia profesional en archivos históricos, la formación en historia sigue siendo indispensable, durante siglos los archiveros provenían de ese campo. En las últimas décadas han proliferado las facultades de Biblioteconomía y Documentación, pero la experiencia nos dice que en dichos grados quizás habría que ampliar el tiempo de dedicación en la formación en historia.

\section{Líneas de trabajo nuevas y prioritarias}


Pienso que los archivos como servicio público que son no pueden permanecer desconectados de lo que sucede en la realidad a la que pertenecen y ser sordos a lo que ocurre en la sociedad en la que vivimos. El archivo debería estar muy atento al latido de la sociedad.

Es un clamor actual en el conjunto de nuestra ciudadanía la necesidad de visibilización de la mujer, opacada a lo largo de la historia. Pero opacar no implica inexistencia, solo ocultamiento, historia velada. En este sentido los archivos tenemos una labor importante que realizar en el conocimiento de la historia de la mujer. Para ello contamos con riquísimos fondos documentales que considero deben ser objeto prioritario de nuestra atención para su puesta a disposición de los investigadores y del público en general, en el menor espacio de tiempo posible. La sociedad está conformada por mujeres y hombres a partes iguales, esta realidad incontestable ha sido ignorada durante siglos, pero a pesar de ello las mujeres han dejado su huella documental, el testimonio de su presencia aparece en infinidad de tipos documentales, el primero y más importante el protocolo notarial. Por tanto, esta presencia, en forma de una gran diversidad de documentos, debe ser rescatada, recuperada y expuesta a luz.

Otro aspecto del latido de la sociedad actual de las últimas décadas es el del activismo social al margen de la tradicional política. Un ejemplo clarísimo de ello es el movimiento $15 \mathrm{M}$, pero ya antes y también después de él, ha sido y es una realidad constante en la vida de nuestro país. Los movimientos ciudadanos al no pertenecer a ámbitos oficiales corren el riesgo de que su huella documental se pierda en un limbo, que el presente no debe permitirse porque falsearía la historia futura. Pienso que es una cuestión importante a plantear en los foros archivísticos.

Acabo ya, en este artículo solo he pretendido realizar una mirada, quizás algo filosófica, sobre nuestra realidad actual extendiéndola y proyectándola hacia el futuro, imaginando lo que quizás estamos llamados a ser, sin dejar de contemplar aquellas zonas que van desapareciendo de nuestro contorno. Mi objetivo ha sido realizar una pequeña reflexión en vOz alta que permita hacernos fantasear un poco sobre un horizonte que puede permitirnos ser lo mejor que podemos ser. 


\section{Bibliografía}

Annual Meeting Council on Public History, 2017. Disponible en: Http://ncph.org/conference/2017-annual-meeting/

El Archivo y documento ante una Nueva Fase de la Revolución Tecnológica. [Congreso] Pamplona, 20 y 21 de junio de 2019. Disponible en: http://www.archiverosdenavarra.org/es/noticia/34/El+Archivo+y+Docu mento + ante + una + Nueva + Fase $+\mathrm{de}+\mathrm{la}+$ Revoluci $\% 260 a c u t e \% 3 \mathrm{Bn}+$ Tecnol $\% 260 a$ cute $\% 3$ Bgica

ESTEPA GIMÉNEZ, Jesús, 1995. El archivo en la enseñanza de la Historia. Revista TRIA, 2, 55-72.

GONZÁLEZ CACHAFEIRO, Javier (Coord.), 2010. 3 as Jornadas Archivando: La difusión en los archivos: Actas de las Jornadas: León, 11 y 12 de noviembre de 2010. León: Fundación Sierra Pambley

HOWARD, Philip N.; JONES, Steve (ed.), 2005. Sociedad on-line/Society Online: Internet En Contexto/The Internet in Context. Barcelona: UOC.

MELERO CASADO, Ana; CRUCES BLANCO, Esther, 1991. Los archivos y los programas pedagógicos. Boletín de la $A N A B A D, 41(2), 33-51$.

MUSEO DEL PRADO, 2019. Tienda. Disponible en: http://www.tiendaprado.com/

NATIONAL COUNCIL ON PUBLIC HISTORY, 2019. Disponible en: http://ncph.org/

OLMO IBÁÑEZ, María del, 2009. Abrir las puertas de los archivos. Revista Archivamos, 70. ISSN: 1576-320X

OLMO IBÁÑEZ, María del, 2010. El latido del archivo de Simancas. Boletín de la Asociación de Archiveros valencianos, 39. ISSN 1578-0538

POVEDA POVEDA, Consuelo; OLMO IBÁÑEZ, María del, 2014. La didáctica en el AHPA. En: El món Educatiu, usuari de l'arxiu per a la recerca. Quartes Jornades Educació $i$ arxius Sant Cugat del Vallès, 27 i 28 de juny de 2013. Barcelona: Institut de Ciències de l'Educació. Universitat, pp. 235 y ss.

Seconda Conferenza Nazionale di Public History AIPH, 2019. Disponible en: https://aiph.hypotheses.org/ 
SIMONET, Julio Enrique, 1997. Recomendaciones para la edificación de archivos. Madrid: Ministerio de Educación, Cultura y Deporte. Subdirección General de Documentación y Publicaciones.

TRIBÓ TRAVERIA, Gemma, 2005. Enseñar a pensar históricamente: los archivos y las fuentes documentales en la enseñanza de la historia. Barcelona: Horsori Editorial. 